\title{
In Silico Screening of Antimicrobial Compounds Using Docked Complexes of Antibiotics and Antimicrobial Peptides
}

\author{
Dinakari Sarangan, Keerthana Sakthivadivelan, Darsini Thiyagarajan, Apsara \\ Sudhakar, Krithika Balakrishnan, Ram Kothandan, and Kumaravel Kandaswamy* \\ ${ }^{1}$ Department of Biotechnology, Kumaraguru College of \\ Technology, Coimbatore - 641049, Tamil Nadu, India.
}

\begin{abstract}
Biofilms are sessile aggregates of bacterial cells enclosed by a slimy matrix that protect the cells from bactericidal molecules. Biofilm associated infections such as Urinary Tract Infections (UTI) are caused by bacterial strains such as Escherichia coli and Enterococcus faecalis. Biofilm often exhibits increased resistance to the antimicrobial compounds due to their polymicrobial nature. The matrix of biofilm consists of exopolysaccharides, extracellular DNA (eDNA), and proteins that are crosslinked to provide structural integrity to the biofilms. The proteins in the biofilm matrix are regarded as the potential targets for the antibiotics and the antimicrobial peptides, which kills the bacterial population in the biofilm by disrupting them. Studies have reported that the metabolically active cells in the biofilms can be killed by antimicrobial peptides while the cells with low metabolic activity can be destroyed by antibiotics. In this study, we have used several combinations of antibiotics and antimicrobial peptides, we have obtained a docked complex of Human Beta Defensin 3 (Positively charged peptide) with Ciprofloxacin (Negatively charged antibiotic) and Dermcidin (Negatively charged peptide) with Tobramycin (Positively charged antibiotic). The efficient pair of antimicrobial peptide and antibiotic was then used to dock with biofilm matrix proteins. In essence, this study aims to provide a combinatorial approach to identify drug targets in biofilm associated infections
\end{abstract}

KEY WORDS: IN-SILICO DOCKING, ANTIMICROBIAL PEPTIDE, BIOFILMS, AND ANTIBIOTICS.

\section{INTRODUCTION}

Pathogenic strains such as Escherichia coli (E.coli) and Enterococcus faecalis (E.faecalis)are the major cause of Urinary Tract Infection (UTI) and other biofilm associated infections(Madrazo et al., 2020)(Govindarajan et al., 2020) In addition, chronic infections such as cystic

\section{ARTICLE INFORMATION}

*Corresponding Author: kumaravel.k.bt@kct.ac.in

Received 05th Oct 2020 Accepted after revision 07th Dec 2020

Print ISSN: 0974-6455 Online ISSN: 2321-4007 CODEN: BBRCBA

Thomson Reuters ISI Web of Science Clarivate Analytics USA and Crossref Indexed Journal

\section{Clarivate
Analytics}

NAAS Journal Score 2020 (4.31) SJIF: 2020 (7.728)

A Society of Science and Nature Publication,

Bhopal India 2020. All rights reserved.

Online Contents Available at: http//www.bbrc.in/

Doi: http://dx.doi.org/10.21786/bbrc/13.11/11 fibrosis and periodontitis were also proven to be biofilms associated infections. In order to establish the infection, the pathogenic bacteria need to attach to the host cells. This host- pathogen interaction leads to primary attachment of bacterial pilus to the host surface and aids in colonizing the host epithelium. Pilus of the bacteria are long filamentous proteins extending from bacterial surfaces. These pilin proteins are the contributory factors for many diseases such as cystitis, meningitis, sepsis, porynephritis and UTI(Sillanpää et al., 2010).

The pilus assembly of gram positive and gram-negative bacteria are very distinct. There are five different types of pilus assembly pathway in gram negative bacteria and those are Chaperone-Usher (CU) pili, type IV pili, type IV secretion pili, type V pili and curli fibres(Guillermo

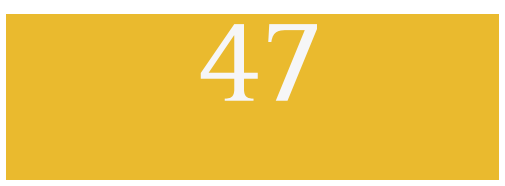


Garcia-Manero Shao-Qing Kuang, Susan 0’Brien, Deborah Thomas, and Hagop Kantarjian, 2005).However, among those pathways CU pili is the most extensively studied pathway. However, in gram positive bacteria, there are only two pathways, one being the well-studied sortase pathway (Telford et al., 2006) and the other the type IV mechanism(Muschiol et al., 2019). Pili in both gram positive and gram negative bacteria is made of major and minor protein subunits. The major pilin subunit is repetitive and more abundant when compared to minor pilin subunits(Giltner, Nguyen and Burrows, 2012).

The Major pilin subunit of CU pili of gram-negative bacteria is fimA and minor pilin subunits are a periplasmic chaperone(fimC), usher (fimD), and a tip adhesion(fimH)(Busch, Phan and Waksman, 2015). In gram positive sortase assembled pili, the major pilin is $\mathrm{EbpC}$ and the minor pilin is adhesion pilin EbpA(La Rosa et al., 2016). Therefore, pili proteins are considered as an attractive target for antimicrobial therapy. Studies in the past demonstrated that Antimicrobial peptides (AMPs) such as human Beta Defensin 3 (hBD3) can focally target sortases and its pili proteins(Kandaswamy et al., 2013). Majority of antibiotics such as ampicillin, tetracycline, streptomycin have been used to treat a wide range of bacterial infections but over a period of time bacterial strains have gained resistance to those antibiotics. Therefore, to overcome this, Antimicrobial peptides (AMPs) were first discovered in the early 1980s and AMPs such Human Beta Defensin 5 (hBD5) were proven to kill bacteria (Chileveru et al., 2015).

In the recent years, AMPs such as dermcidin were also proved to be act against pathogens(Schittek et al., 2001), however the bacterial strains acquired resistance to those AMPs making it challenging to treat bacterial infections(Schmidtchen et al., 2002).Therefore, in this study, we have used several combinations of antibiotics and antimicrobial peptides. We have obtained a docked complex of Human Beta Defensin 3 (Positively charged peptide) with Ciprofloxacin (Negatively charged antibiotic) and Dermcidin (Negatively charged peptide) with Tobramycin (Positively charged antibiotic). Furthermore, this study also demonstrate that the biofilm associated pili protein (FimA) can be targeted using docked complexes of AMPs and antibiotics. (yen and Burrows, 2012) The Major pilin subunit of CU pili of gram-negative bacteria is fimA and minor pilin subunits are a periplasmic chaperone(fimC), usher (fimD), and a tip adhesion(fimH)(Busch, Phan and Waksman, 2015).

\section{MATERIAL AND METHODS}

Target Selection: The target selection was performed as mentioned in previous studies (Table:1). We have chosen few well studied antibiotics and AMP for docking as mentioned in Table 1.

Retrieval and Preparation of target protein: The crystallized structure of the antimicrobial proteins were retrieved from Protein Data Bank (PDB) andthe energy minimization of proteins was performed using GROMACS(Lemkul, 2019). Then protein was prepared using the protein preparation as mentioned in the previous studies(Madhavi Sastry et al., 2013) the auto dock software assigns missing bonds, bond order, flexible torsions and charges to the input structures during the preparation process and makes them readily available for docking studies(Sivaramakrishnan et al., 2019)

Retrieval and Preparation of ligands: The well-studied antibiotics (as shown in Table 1) and its 3-Dimensional structure was retrieved from the Drug bank and prepared for docking studies. An autodock user module 4.2 was used in this study. The auto dock software assigns missing charges, bonds, bond order and hybridization, detects flexible torsions, creates explicit hydrogens and finally energy-minimized structure can be obtained(Sivaramakrishnan et al., 2019).

Molecular Docking: A blind docking was performed using autodock vina as described in the previous study (Sivaramakrishnan et al., 2019). Molecular docking was performed to understand the interaction of selected AMP's with antibiotics . The Initial docking analysis was performed using the autodock 4.2 package (Sivaramakrishnan et al., 2019). The surface module of autodock creates a double colored molecular surface according to the electrostatic property of the receptor protein. The cavity prediction algorithm predicts the cavities present in the receptor protein and displays it to the user in green color and finds the potential binding sites of the receptor protein. The parameters were set to a molecular surface with extended Van der Waals and number of cavities to five.

The docking was carried out using autoDock simplex evolution search algorithm with grid resolution $30 \AA$ for grid generation and cavity predicted using a search algorithm called cavity prediction algorithm.43 In cavity prediction wizard the number of cavities was restricted to three and the cavity with the large volume was selected as the origin for the binding site. The docking wizard runs with default parameters autoDock as a search algorithm, number of runs, maximum population and maximum iteration was limited to 10,50 and 1500 respectively. The selected phytochemicals were docked against the receptor proteins and best-generated poses were selected based on the docking scores. The Interaction between the ligand and the receptor protein depends on the number of H-bonds, distance and binding energy. Some poses have favorable hydrogen bond interactions with active site amino acid residues of target bacterial membrane proteins.(Sivaramakrishnan et al., 2019).

\section{RESULTS AND DISCUSSION}

Combinational therapy is a promising approach to overcome and mitigate antimicrobial resistance. In combinational therapy, a combination of conventional antibiotics is used together with other antimicrobial peptides to increase the treatment efficacy (Thappeta et al., 2020). Combinational therapy can extend the 
lifetime of drugs, inhibits after effects and mitigates the emergence of resistance. While there have been several reports of synergy between conventional antibiotics and other drugs, very few have examined synthetic antimicrobial peptides in combination with conventional antibiotics(Thappeta et al., 2020). In this study, we have docked several antimicrobial peptides and antibiotics to obtain a docked complex of opposite charges (Figure 1 \& Table 1) using auto dock vina. The docking score represents the affinity of the antibiotics towards the antimicrobial peptide. More negative the docking score, better the binding affınity.

Table1. Proteins and AMP's chosen for docking

\begin{tabular}{|c|c|c|c|c|c|c|}
\hline Peptides & PDB ID & Charge & Ligand & Drug bank ID & Charge & References \\
\hline HBD-3 & $1 \mathrm{KJ} 6$ & Positive & Ciprofloxacin & DB00537 & Negative & $\begin{array}{l}\text { (Dhople, Krukemeyer and } \\
\text { Ramamoorthy, 2006) (Walters et al., 2003) }\end{array}$ \\
\hline Dermcidin & 2YMK & Negative & Tobramycin & DB00684 & Positive & (Schittek et al., 2001) (Walters et al., 2003) \\
\hline Hevein & 1Q9B & Negative & Streptomycin & DB01082 & Positive & $\begin{array}{c}\text { (Prabhu et al., 2013)(Tseng, Bryan and } \\
\text { Van den Elzen, 1972) }\end{array}$ \\
\hline LL 37 & $2 \mathrm{~K} 60$ & Positive & Tetracycline & DB00759 & Negative & (Overhage et al., 2008)(Pamp et al., 2008) \\
\hline
\end{tabular}

Table 2. Estimation of docking scores using autodock vina

\begin{tabular}{|l|c|}
\hline Protein and Ligand Complex & Docking score \\
\hline $\begin{array}{l}\text { Human Beta Defensin 3 } \\
\text { with Ciprofloxacin }\end{array}$ & -5.0 \\
\hline Dermcidin with Tobramycin & -5.2 \\
\hline Hevein with Streptomycin & -5.2 \\
\hline LL-37 with Tetracycline & -5.6 \\
\hline
\end{tabular}

Table 3. Scores of FimA docked with Antibiotics

\begin{tabular}{|l|c|}
\hline Antibiotics & Scores \\
\hline ampicillin & -5.6 \\
\hline ciprofloxacin & -5.7 \\
\hline streptomycin & -5.6 \\
\hline tobramycin & -5.2 \\
\hline Tetracycline & -5.9 \\
\hline
\end{tabular}

Table 4. Docking score of protein-protein docking complexes

\begin{tabular}{|l|c|c|}
\hline s.no & $\begin{array}{c}\text { Protein complexes } \\
\text { (pilin protein-AMPs) }\end{array}$ & z-score \\
\hline 1 & FimA-Havein & -1.4 \\
\hline 2 & FimA-LL37 & -1.8 \\
\hline 3 & FimA-HBD3 & -2.1 \\
\hline
\end{tabular}

The complex Dermcidin and tobramycin with an affinity of-5.2(Table 2) has the highest affinity as Tobramycin is docked with ASP 42, ASP 45 ,SER 46 which creates an ionic interaction. Also the shorter distance $(<3 \AA)$ between the peptide and the antibiotic can be clearly seen
Figure 1: Docked image of (a)Ciprofloxacin and Human beta defensin 3 (b)Tobramycin-and Dermcidin (c)Hevein and Streptomycin (d)LL-37 and Tetracycline

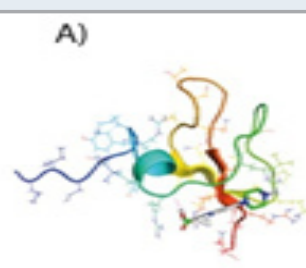

B)

C)

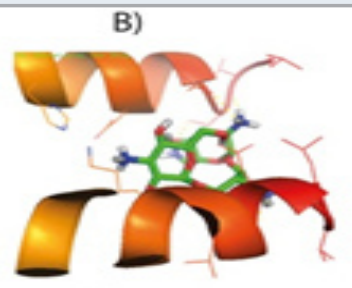

D)

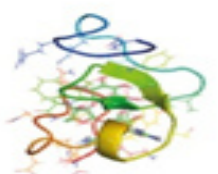

Figure 2: Ligplot image of docked complexes of (a) Ciprofloxacin and Human beta defensin 3 (b)Tobramycin and Dermcidin (c) Hevein and Streptomycin (d) LL-37 and Tetracycline

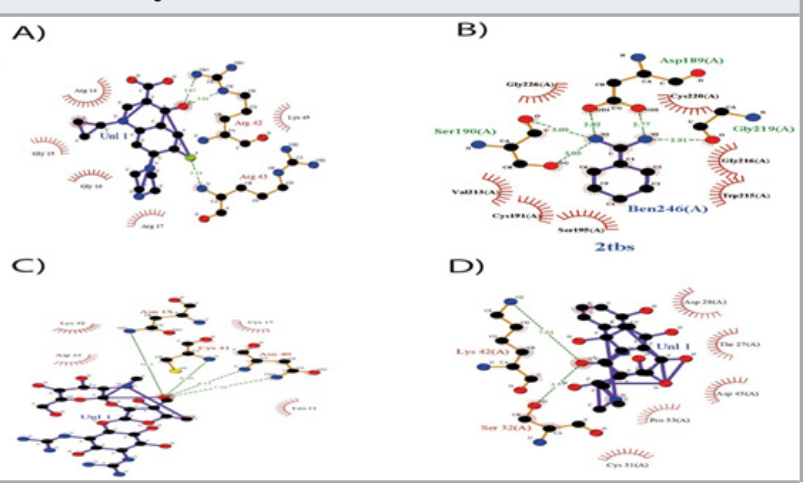

in the ligplot result (Figure 2 b).From the affinity scores of FimA and antibiotics complexes (as mentioned in Table 3) it is evident that fimA has a higher affinity of -5.9 for tetracycline which can be seen in the ligplot results (Figure 3e) . A 3D image of FimA and antibiotics with 
hydrogen bonds can be seen in figure 4 . The antibiotic ampicillin form hydrogen bonds with TYR 158, LYS68 (of FimA), Ciprofloxacin form hydrogen bonds with TYR158, SER67, LYS68 , streptomycin form hydrogen bonds with ASP29, GLY26, GLY53, ASN55, THR31, tobramycin form hydrogen bonds with GLN33, THR31, GLN30, GLY26, SER27, ASN 55, and tetracycline form hydrogen bonds with GLN98, THR9 of FimA can be seen in ligplot results (Figure 3).

Figure 3: Ligplot image of docked complexes a) fimA and ampicillin b) fimA and ciprofloxacin c) fimA and streptomycin d) fimA and Tobramycin e) fimA and tetracycline.

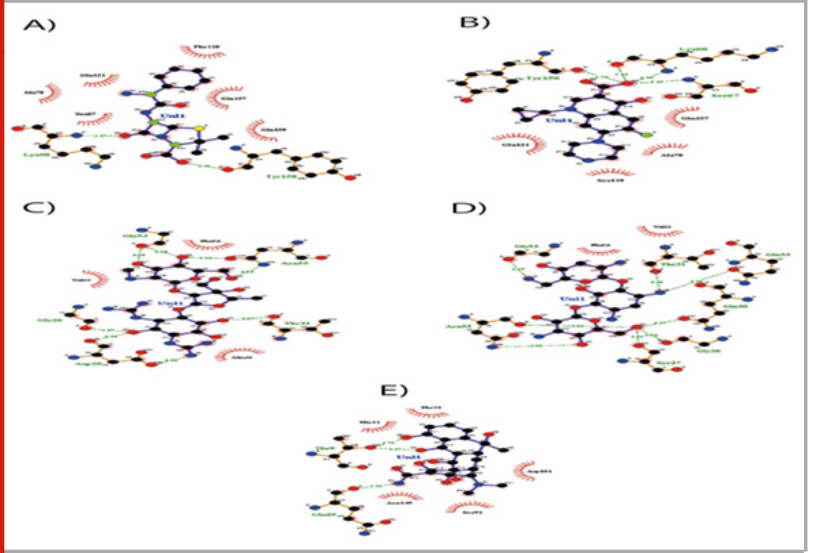

Figure 4: Docked images of fimA with antibiotics a) fimAampicillin b) fimA-ciprofloxacin c) fimA-streptomycin d) fimA-Tobramycin e) fimA- tetracycline

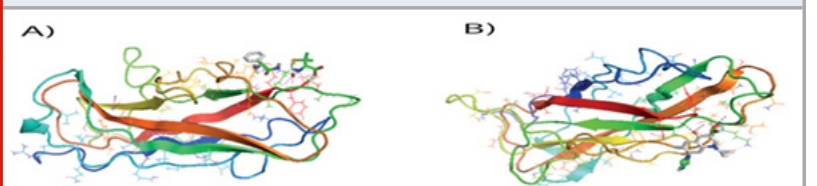

c)

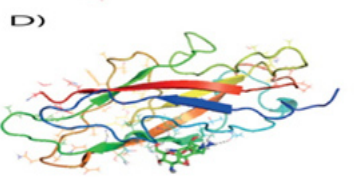

E)

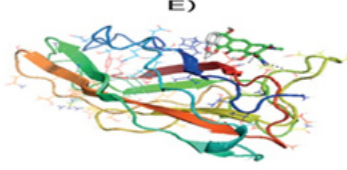

Protein - protein Docking was carried out using HADDOCK online tool (Van Zundert et al., 2016) Based on $\mathrm{Z}$ score the best docked complex was chosen among clusters. The best docked complexes were chosen for all the antimicrobial peptides with FimA listed in the Table 4. Dimplot and PIC: Protein Interactions Calculator(Tina, Bhadra and Srinivasan, 2007) were used to analyze the interactions between FimA and antimicrobial peptides. The Important interactions between FimA and AMPs based on the bond length are ASP62(A):TYR9(B),ALA 25(A):ARG36(B), VAL123(A):THR35(B) of Human beta defensin(B), LYS155(A):GLN29(B) of hevein,andGLU15
Figure 5: Docked of fimAwith Anti-microbial peptides a) fimA-Hevein b) fimA- Human beta defensin c) fimALL-37

A)

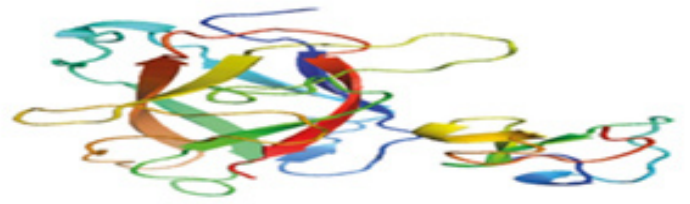

B)

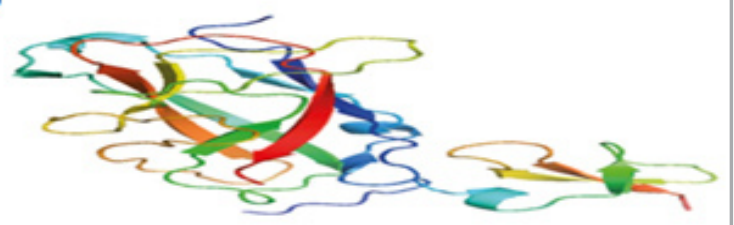

C)

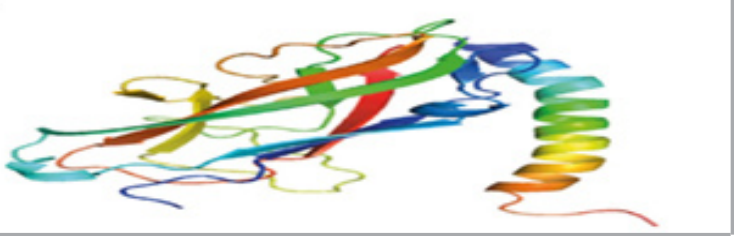

Figure 6: Dimplot results of FimA docked with Antimicrobial peptides.chainA -FimA, chainB-AMPs a)fimA-HBD3 b) fimA- Hevein c) fimA-LL-37

A)

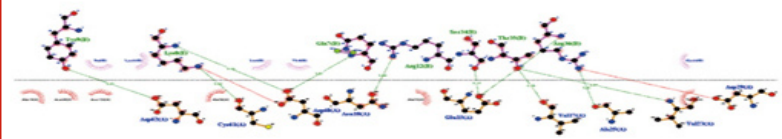

B)
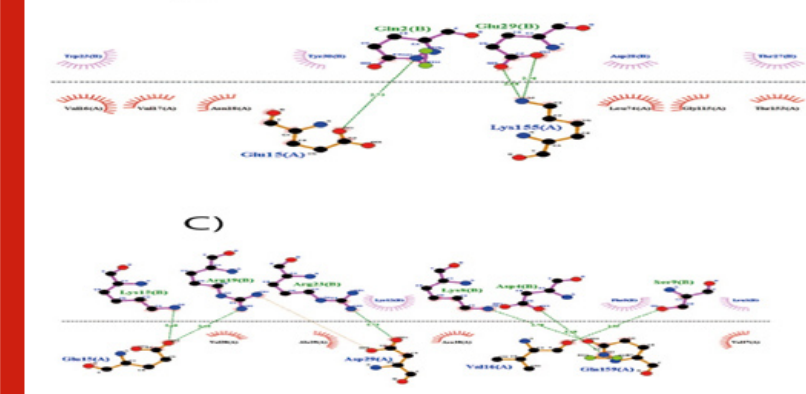

(A):LYS15(B),ARG19(B),of LL-37. it can be seen in the Dimplot(Figure 6).

\section{CONCLUSION}

The best docked complex is dermcidin and tobramycin with an affinity of - 5.2 and FimA with tetracyclin with an affınity of -5.9. This study has few limitations. One limitation of this study is that molecular level analysis of the docked complexes cannot be done since high resolution techniques such as X-ray crystallography should be done to verify the in-vivo complex formation of AMPs and antibiotics. The other limitation is, that electrophoresis technique is required to verify the increase in the molecular weight of docked complexes. 
In addition, further experimental investigation is required to verify the binding of already docked complexes with matrix polysaccharides and proteins.

\section{REFERENCES}

Busch, A., Phan, G. and Waksman, G. (2015) 'Molecular mechanism of bacterial type 1 and $\mathrm{P}$ pili assembly', Philosophical Transactions of the Royal Society A: Mathematical, Physical and Engineering Sciences, 373(2036). doi: 10.1098/rsta.2013.0153.

Chileveru, H. R. et al. (2015) Visualizing attack of escherichia coli by the antimicrobial peptide human defensin 5, Biochemistry. doi: 10.1021/bi501483q.

Dhople, V., Krukemeyer, A. and Ramamoorthy, A. (2006) 'The human beta-defensin-3, an antibacterial peptide with multiple biological functions', Biochimica et Biophysica Acta - Biomembranes. doi: 10.1016/j. bbamem.2006.07.007.

Giltner, C. L., Nguyen, Y. and Burrows, L. L. (2012) 'Type IV Pilin Proteins: Versatile Molecular Modules', Microbiology and Molecular Biology Reviews, 76(4), pp. 740-772. doi: 10.1128/mmbr.00035-12.

Govindarajan, D. K. et al. (2020) 'Adherence patterns of Escherichia coli in the intestine and its role in pathogenesis', Medicine in Microecology. doi: 10.1016/j. medmic.2020.100025.

Guillermo Garcia-Manero Shao-Qing Kuang, Susan O'Brien, Deborah Thomas, and Hagop Kantarjian, H. Y. (2005) 'NIH Public Access', Bone, 23(1), pp. 1-7. doi: 10.1016/j.tim.2010.03.002.A.

Kandaswamy, K. et al. (2013) 'Focal targeting by human $\beta$-defensin 2 disrupts localized virulence factor assembly sites in Enterococcus faecalis', Proceedings of the National Academy of Sciences of the United States of America, 110(50), pp. 20230-20235. doi: 10.1073/ pnas. 1319066110.

La Rosa, S. L. et al. (2016) 'Enterococcus faecalis ebp pili are important for cell-cell aggregation and intraspecies gene transfer', Microbiology (United Kingdom), 162(5), pp. 798-802. doi: 10.1099/mic.0.000276.

Lemkul, J. (2019) 'From Proteins to Perturbed Hamiltonians: A Suite of Tutorials for the GROMACS2018 Molecular Simulation Package [Article v1.0]', Living Journal of Computational Molecular Science. doi: 10.33011/livecoms.1.1.5068.

Madhavi Sastry, G. et al. (2013) 'Protein and ligand preparation: Parameters, protocols, and influence on virtual screening enrichments', Journal of ComputerAided Molecular Design. doi: 10.1007/s10822-0139644-8.

Madrazo, M. et al. (2020) 'Predictive factors for
Enterococcus faecalis in complicated communityacquired urinary tract infections in older patients', Geriatrics and Gerontology International, 20(3), pp. 183-186. doi: 10.1111/ggi.13856.

Muschiol, S. et al. (2019) 'Gram-Positive Type IV Pili and Competence', Protein Secretion in Bacteria, pp. 129-135. doi: 10.1128/microbiolspec.psib-0011-2018. Overhage, J. et al. (2008) 'Human host defense peptide LL-37 prevents bacterial biofilm formation', Infection and Immunity. doi: 10.1128/IAI.00318-08.

Pamp, S. J. et al. (2008) 'Tolerance to the antimicrobial peptide colistin in Pseudomonas aeruginosa biofilms is linked to metabolically active cells, and depends on the pmr and mexAB-oprM genes', Molecular Microbiology. doi: 10.1111/j.1365-2958.2008.06152.x.

Prabhu, S. et al. (2013) 'Anionic Antimicrobial and Anticancer Peptides from Plants', Critical Reviews in Plant Sciences. doi: 10.1080/07352689.2013.773238.

Schittek, B. et al. (2001) 'Dermcidin: A novel human antibiotic peptide secreted by sweat glands', Nature Immunology. doi: 10.1038/ni732.

Schmidtchen, A. et al. (2002) 'Proteinases of common pathogenic bacteria degrade and inactivate the antibacterial peptide LL-37', Molecular Microbiology. doi: 10.1046/j.1365-2958.2002.03146.x.

Sillanpää, J. et al. (2010) 'Characterization of the ebpfm pilus-encoding operon of enterococcus faecium and its role in biofilm formation and virulence in a murine model of urinary tract infection', Virulence. doi: 10.4161/viru.1.4.11966.

Sivaramakrishnan, M. et al. (2019) 'Screening of curcumin analogues targeting Sortase A enzyme of Enterococcus faecalis: a molecular dynamics approach', Journal of Proteins and Proteomics. doi: 10.1007/ s42485-019-00020-y.

Telford, J. L. et al. (2006) 'Pili in Gram-positive pathogens', Nature Reviews Microbiology, 4(7), pp. 509-519. doi: 10.1038/nrmicro 1443.

Thappeta, K. R. V. et al. (2020) 'Combined Efficacy of an Antimicrobial Cationic Peptide Polymer with Conventional Antibiotics to Combat MultidrugResistant Pathogens', ACS infectious diseases, 6(5), pp. 1228-1237. doi: 10.1021/acsinfecdis.0c00016.

Tina, K. G., Bhadra, R. and Srinivasan, N. (2007) 'PIC: Protein Interactions Calculator', Nucleic Acids Research, 35(SUPPL.2), pp. 473-476. doi: 10.1093/nar/gkm423. Tseng, J. T., Bryan, L. E. and Van den Elzen, H. M. (1972) 'Mechanisms and spectrum of streptomycin resistance in a natural population of Pseudomonas aeruginosa.', Antimicrobial agents 